\title{
Flexibility analysis on a supply chain contract using a Parametric Linear Programming Model
}

\author{
Chengbin Chu*, Eric E. Longomo, Xiang Song and Djamila Ouelhaj
}

\begin{abstract}
This research paper builds on existing knowledge in the field of parametric Linear Programming $(p L P)$ and proposes a continuous mathematical model that considers a multi-period Quantity Flexibility (QF) contract between a car manufacturer (buyer) and external parts supplying company. The supplier periodically delivers parts to the car manufacturer as agreed in the contract. Due to the uncertainty of the demand for parts, the car manufacturer -in concert with the supplieraims to develop a policy -at strategic level, that determines the optimal nominal order quantity $(Q)$ and variation rate $(\beta)$ underpinning the contract that ensures the actual order quantity satisfies the actual demand and the total cost is minimised over the contract length. The behaviour of the mathematical model has been examined in order to establish its feasibility and convexity, consequently guaranteeing an optimal solution. Simulations have been carried out to evaluate the relationship of the total cost with respect to the variation rate and the nominal quantity ordered.
\end{abstract}

Keywords: multi-period, quantity flexibility, parametric linear programming, contracting

\section{INTRODUCTION}

Supply chain (SC) coordination through contracts has been widely studied in literature and extensively used in industries. Detailed reviews of SC coordination through contracts are given in the works of [(Cachon, 2003); (Whang, 1995); (Lariviere, 1999); (Tsay et al., 1999b)]. Although the three types of flows -material, information and financial are well known, Hohn (2010) argues that classifying Supply chain contracts is not straightforward.

In this paper, the literature review's focus is placed on contract flexibility -frequently used in capacity reservation for transportation and also similar works are found in high tech industries, such as automotive parts and semiconductor (Knoblich et al., 2011). These industries are brought to carefully consider the way their businesses are conducted due to their rapidly changing technological realm, capital intensive investment approach and high demand uncertainty [(Knoblich et al., 2011), (Park and Kim, 2013)]. To overcome these hurdles, flexible supply coordination through contracts between the partners is commonly used.

* Chengbin Chu is with Laboratoire Génie Industriel, Ecole Centrale Paris, Grande Voie des Vignes 92295, Grande Voie des Vignes 92295, ChâtenayMalabry Cedex, Paris, France.

Eric E. Longomo, Xiang Song and Djamila Ouelhaj are with University of Portsmouth, Department of Mathematics, Lion Gate Building, Lion Terrace, Hampshire, Portsmouth (UK), PO1 3HF
According to Das and Abdel-Malek (2003), SC flexibility is the robustness of the buyer-supplier relationship under changing supply conditions.

There has been growing body of research related to the literature of QF. These are split into two major taxonomic groups (Park and Kim, 2013). The first consist of: general contracts -commonly found in manufacturing and retail industries, addressing contractual clauses including pricing, Buy-Back or Return Policies, Quantity Flexibility (QF), Minimum Commitment (MC), Allocation Rules (AR) and Lead time. Under this group of clauses, the flexibility allows some deviation in the buyer ultimate procurement. The second encompasses specialised contracts, commonly employed in capital intensive industries (Park and Kim, 2013).

Sethi et al (2004) carried out work on both single and multiperiod versions of quantity flexibility contracts that considered a single demand forecast update per period and a spot market. Sethi et al (2004) modelled the problem as a one period, two stage quantity flexibility contracts between a buyer and a supplier and then as a multi-period stochastic dynamic programing problem utilising stochastic comparison theory to investigate the effect on the optimal policy and the expected profit of the quality of forecast updates. Their work culminated to methods that allowed obtaining an optimal order quantity from a contracted supplier and a spot market. Kim (2011) studied the effects of QF contract on the performance of a two-echelon supply chain under dynamic market demands. Kim (2011) analysed the flexibility profile of the QF contract stemming from a discrete-event simulation approach that was aimed at comparing the impacts of the given order policy on performance outcome with and without the QF contract.

Contrary to previous similar works where the optimal nominal quantity and flexibility parameters were predicted using solely deterministic and probabilistic models, this current work considers a deterministic setting of forecast or historical requirement for a "one year finite horizon" and extend the projection accounting for the case where the data in the objective could be continuous by fitting a $p L P$ model. Hence forth we propose, in this work, a finite horizon $p L P$ model that considers a quantity flexibility contract between two independent players. A car manufacturer, a Stackelberg leader and a parts supplying company working together in order to minimise -at the strategic level, the standard deviation between ultimate parts procurement and the nominal order quantity $(Q)$ initially placed by the car 
manufacturer. This feat is accomplished by minimising the order flexibility -which translates in practice to the minimisation of the variation rate $(\boldsymbol{\beta})$. A natural constraint of this exercise is that the optimal order quantity in each period in the planning horizon is restricted within the minimum and maximum order quantity level. The collaboration between the two players will amount to incentives on both parties in the form of reduced uncertainty and optimum ordering cost for the supplier and the car manufacturer respectively.

\section{MODEL DEVELOPMENT}

The model considered in the current work is an example of a two-echelon SC, in which a QF contract is agreed between two main players, a buyer and supplier. The buyer is provided with some flexibility with respect to the nominal ordering quantity $Q$ but, is duty bound to commit to minimum purchase quantity, $L(\beta)$, below the initial order. The supplier in return, agrees to meet the actual order quantity (or firm order) provided that it falls below the maximum allowable purchase quantity, $U(\beta)$ above the nominal quantity. The supplier charges a unit purchasing cost $p(\beta)$ to contain risks. When signing the contract with the supplier, $\beta$ and $Q$ need to be decided to minimise the total cost. This problem is a big challenge to the buyer due to the high variation of the actual demand.

\section{A. Notations}

The following notations will be used throughout this paper.

i. Input Data

$T \quad$ Number of periods in the contracts, thus period, $t=1,2, \ldots, T$, represents different periods within the planning horizon

$d_{t} \quad$ Demand at time $t$ (unknown in reality. In this paper, demand is forecasted using historical data.)

$h \quad$ Unit inventory holding cost per period

$s \quad$ Unit shortage cost per period

ii. Decision Variables

$x_{t} \quad$ Order quantity of period t, $x=\left(x_{1}, \ldots, x_{T}\right)$

$\beta \quad$ Variation rate with respect to the nominal quantity $(Q)$

$Q \quad$ Nominal order quantity

$p(\beta) \quad$ Unit purchasing cost in function of the variation rate. Assumption is made in this current work that $p(\beta)$ is a linear or piecewise linear convex function

$U(\beta) \quad$ Upper bound on ordered quantity per period, where $U(\beta)=Q(1+\beta) \geq x_{t}$

$L(\beta) \quad$ Lower bound on the ordered quantity per period, where $L(\beta)=Q(1-\beta) \leq x_{t}$

$v_{h s} \quad$ Total holding /shortage cost

$K_{t} \quad$ Purchasing cost at period $t$

$g(\beta, Q, x)$ Total cost over the length of the contract

\section{B. Cost Analysis}

In each period of the contract, three costs will be incurred Purchasing cost, inventory cost and holding cost. The total cost is thus defined as the sum of these three costs. With different order quantities in each period, the cost will be different.

The purpose of the analysis is to determine the optimal values of $\boldsymbol{\beta}$ and $\boldsymbol{Q}$ that minimises the deviation between the initial and ultimate procurement, consequently minimising the total cost of purchase, inventory holding and shortage costs.

Assumptions are made that:

- All current or back ordered demands need to be satisfied at the end of the contract meaning that no ordering cost is incurred.

- The unit purchase cost $p(\beta)$ is assumed to be linear or piecewise linear convex function and is given by the expression: $p(\beta)=c_{0}+\beta \cdot c_{1}$

Where $c_{0}$, represents the minimal possible cost with zero flexibility and $c_{1}$ is a given fixed rate of change of $p(\beta)$.

\section{Construction of the cost function}

If $d_{1}, \ldots, d_{T}$ are the demands for the next $\mathrm{T}$ periods and backorder is allowed, two cases arise:

1. Holding/shortage cost for period $t\left(v_{t}\right)$

$h \cdot \sum_{i=1}^{t}\left(x_{i}-d_{i}\right)$ If $\sum_{i=1}^{t}\left(x_{i}-d_{i}\right) \geq 0$

$s \sum_{i=1}^{t}\left(d_{i}-x_{i}\right)$ If $\sum_{i=1}^{t}\left(x_{i}-d_{i}\right) \leq 0$

This leads to the following

$v_{t}=\max \left[h \sum_{i=1}^{t}\left(x_{i}-d_{i}\right), s \sum_{i=1}^{t}\left(d_{i}-x_{i}\right)\right]$

2. Purchasing Cost for period $t\left(K_{t}\right)$

$K_{t}=p(\beta) \cdot x_{t}$

3. The total cost can then be written as:

$f(\beta, Q, x)=\sum_{t=1}^{T}\left(v_{t}+K_{t}\right)$

\section{Problem formulation}

In this paper, we consider that backorder is allowed.

The optimisation problem can be formulated as:

Minimize: $f(\beta, Q, \boldsymbol{x})$

$x_{t} \geq Q(1-\beta), \quad t=1,2, \ldots, T$

$-x_{t} \geq-Q(1+\beta), \quad t=1,2, \ldots, T$

$1 \geq \beta \geq 0$

$Q \geq 0$

Given the vector of order quantity, $\boldsymbol{x}^{*}(\beta, Q)=\operatorname{argmin}\{f(\beta, Q, \boldsymbol{x}) \mid$ $\left.Q(1-\beta) \leq x_{t} \leq Q(1+\beta), t=1, \ldots, T\right\}$, and $g(\beta, Q)=f\left(\beta, Q, \boldsymbol{x}^{*}(\beta, Q)\right)$. 
Our problem is to find the values of $\beta$ and $Q$ minimizing $g(\beta, Q)$ such that $1 \geq \beta \geq 0$ and $Q \geq 0$.

\section{LINEARIZATION OF THE MODEL}

The objective function (6) in Section II is nonlinear. To linearize the objective function, we introduce the additional decision variable and addition constraints as follows:

- $J_{t}:$ The inventory holding/shortage cost of period $t, t=1,2, \ldots, T$

- $J_{t}-h \sum_{i=1}^{t} x_{i} \geq-h \cdot D_{t}, t=1,2, \ldots, T$

- $J_{t}+s \sum_{i=1}^{t} x_{i} \geq s \cdot D_{t} \quad t=1,2, \ldots, T$

Where $D_{t}=\sum_{i=1}^{t} d_{i}$ represents the cumulative demand from initial to current period.

- Assumption is made, without being restrictive, that $\sum_{t=1}^{T} x_{t}=\sum_{t=1}^{T} d_{t}=D_{T} \quad, \quad$ making $\quad \sum_{t=1}^{T} K_{t}=$ $\sum_{t=1}^{T} p(\beta) \cdot x_{t}=p(\beta) \cdot D_{T}$ independent of $x_{t}$ and hence can be dropped from (6) when computing $x^{*}(\beta, Q)$ and from the optimisation process.

- $\quad$ Define $y_{t}=x_{t}-Q(1-\beta), t=1,2, \ldots, T$

Applying the above substitutions and assumptions to the initial mathematical model (6) - (8), we have the Primal $p L P$ expressed by:

Minimise $\sum_{t=1}^{T} J_{t}$

s.t:

$$
\begin{array}{ll}
\text { - } & J_{t}-h \sum_{i=1}^{t} y_{i} \geq h\left[-D_{t}+t Q(1-\beta)\right] \\
& t=1,2, \ldots, T \\
\text { - } & J_{t}+s \sum_{i=1}^{t} y_{i} \geq s\left[D_{t}-t Q(1-\beta)\right], \\
& t=1,2, \ldots, T \\
\text { - } & -y_{t} \geq-2 Q \beta \\
& t=1,2, \ldots, T \\
\text { - } & \sum_{t=1}^{T} y_{t}=D_{T}-T Q(1-\beta) \\
\text { - } & y_{t} \geq 0 \\
& t=1,2, \ldots, T
\end{array}
$$

By introducing $\boldsymbol{\varepsilon}, \eta, \boldsymbol{\theta}$ and $\mathbf{q}$ as the multipliers of the constraints (13) - (16) respectively, and letting:

$\Delta_{t}=D_{t}-t Q(1-\beta)$

$t=1,2, \ldots, T$

The dual $p L P$ of the primal $p L P$ can be written as follows:

$$
\begin{aligned}
& t=1,2, \ldots, T \\
& \text { S.t: } \\
& -h \sum_{i=t}^{T} \varepsilon_{i}+s \sum_{i=t}^{T} \mathrm{\eta}_{i}-\theta_{t}+q \leq 0 \\
& \quad t=1,2, \ldots, T \\
& \varepsilon_{t}+\mathrm{\eta}_{t}=1 \\
& \quad t=1,2, \ldots, T \\
& \varepsilon_{t}, \mathrm{y}_{t}, \theta_{t} \geq 0
\end{aligned}
$$$$
\operatorname{Max} \Delta_{T} q+\sum_{t=1}^{T}\left[-h \cdot \Delta_{t} \varepsilon_{t}+s \cdot \Delta_{t} \eta_{t}-2 Q \beta . \theta_{t}\right]
$$

Let $v(\beta, Q)$ be the optimal value of the objective function of this (primal or dual) LP, then: $g(\beta, Q)=p(\beta) D_{T}+v(\beta, Q)$

Note that the first term, which is independent of $\mathrm{x}$, has been dropped from the LP, and must be reintroduced when computing $g$.

\section{THEORETICAL ANALYSIS}

Notice that the primal $\mathrm{pLP}$ is a Right-hand-side $p L P$ (RHS$p L P$ ) of parameters $\beta$ and $Q$ and its dual is an "objective function $p L P$ " $(O F-p L P)$. The examination of the behaviour of the objective function is thus less complex using the dual $p L P$. Since the examination of the joint convexity property of $g(\beta, Q)$ with respect to both $\beta$ and $Q$ is complicated. We leave this proof for our research work.

In this paper, we are to examine the joint convexity property of $g(\beta, Q)$ by some simulation work. To validate our simulation process, we provide the proof of the convexity of $g(\beta, Q)$ by fixing either $\beta$ or $Q$ first.

Theorem 1: Given a fixed value of $\mathrm{Q}, g(\beta, Q)$ is a convex function with respect to parameter $\beta$.

Proof: Since from (22), we know that $g(\beta, Q)=p(\beta) D_{T}+$ $v(\beta, Q)$ and $p(\beta) D_{T}$ are a convex function with respect to $\beta$ obviously, we just need to prove that $v(\beta, Q)$ is a convex function with respect to $\beta$. Since $\mathrm{Q}$ is fixed we use $v(\beta)$ to replace $v(\beta, Q)$ in the following discussion.

Let $\boldsymbol{\varepsilon}, \mathrm{\eta}$ and $\boldsymbol{\theta}$ be $\mathrm{T}$-vectors such that:

$\boldsymbol{\varepsilon}=\left(\varepsilon_{1}, \ldots, \varepsilon_{T}\right)^{\boldsymbol{T}}, \eta \mathrm{\eta}=\left(\mathrm{\eta}_{1}, \ldots, \mathrm{\eta}_{T}\right)^{\boldsymbol{T}}, \quad \boldsymbol{\theta}=\left(\theta_{1} \ldots, \theta_{T}\right)^{\boldsymbol{T}}$ and $\boldsymbol{q}=(q, \ldots, q)^{\boldsymbol{T}}$ a unit vector, the solution vector of the dual $P L P$ can be written as $\boldsymbol{S}=(\boldsymbol{q}, \boldsymbol{\varepsilon}, \eta, \boldsymbol{\theta})$

The objective function $v(\beta)$ can be written as $(\beta)=\boldsymbol{U}(\beta)$. $\boldsymbol{s}$, where $\boldsymbol{U}(\beta)=[(\boldsymbol{c}+\boldsymbol{\gamma} \beta)]$ is the coefficient vector.

We can assume without loss of generality that the dual $p L P$ problem can be expressed as:

$$
v(\beta)=\max \left\{\boldsymbol{U}(\beta) . \boldsymbol{S} \mid \boldsymbol{A} . \boldsymbol{S} \leq \boldsymbol{Y}, \boldsymbol{S} \geq 0, \boldsymbol{A} \in R^{m \times n}, \beta \in\right.
$$

With $\boldsymbol{A}$ being the matrix of the coefficients of the constraints, $\boldsymbol{Y}$, being the set of vectors containing the right hand side of the constraints, and $S$, the set of vectors containing the decision vectors of the standard $p L P$ problem.

The feasibility of $v(\beta)$ is clearly independent of the objective function, hence forth, only the case when the problem is feasible is addressed.

- Let $0 \leq \beta \leq 1$, be the range of values of $\beta$ for which a finite maximum exists for $v(\beta)$

- Let $\beta_{1}$ and $\beta_{2}$, be any two points in the interval $[0,1]$ such that $\boldsymbol{s}_{\mathbf{1}}^{*}$ and $\boldsymbol{s}_{\mathbf{2}}^{*}$ are the corresponding optimal solutions to the dual LP with objective functions $v\left(\beta_{1}\right)$ and $v\left(\beta_{2}\right)$. 
- $\forall \boldsymbol{\alpha} \in[\mathbf{0 , 1}]$, we define $\beta_{3}=\alpha \cdot \beta_{1}+(1-\alpha) \cdot \beta_{2}$ and let the optimal solution for $v\left(\beta_{3}\right)$ be $\boldsymbol{s}_{\mathbf{3}}^{*}$ and $v^{*}\left(\beta_{3}\right)$ be its optimal objective value

$$
\begin{aligned}
v^{*}\left(\beta_{3}\right) & =U\left(\beta_{3}\right) \cdot s_{3}^{*} \\
& =\left(\boldsymbol{c}+\gamma \beta_{3}\right) \cdot s_{3}^{*} \\
& =\left[\boldsymbol{c}+\left(\alpha \cdot \beta_{1}+(1-\alpha) \cdot \beta_{2}\right) \cdot \gamma\right] \cdot s_{3}^{*} \\
& =\alpha \cdot\left(\boldsymbol{c}+\beta_{1} \cdot \gamma\right) \cdot \boldsymbol{s}_{3}^{*}+(1-\alpha) \cdot\left(\boldsymbol{c}+\beta_{2} \cdot \gamma\right) \cdot s_{3}^{*} \\
& \leq \alpha \cdot v^{*}\left(\beta_{1}\right)+(1-\alpha) \cdot v^{*}\left(\beta_{2}\right)
\end{aligned}
$$

The above inequality holds since:

- $\boldsymbol{s}_{3}^{*}$ is feasible solution to the Dual pLP with objective functions $v\left(\beta_{1}\right)$ and $v\left(\beta_{2}\right)$.

Thus given a fixed value of $Q, v(\beta, Q)$ is a convex function with respect to parameter $\beta$. And in general, the theorem 1 holds.

Corollary 1: Given a fixed value of $\beta, g(\beta, Q)$ is a convex function with respect to parameter $Q$.

\section{COMPUTATIONAL RESULTS}

We are to explore the convexity of $g(\beta, Q)$ with respect to $\beta$ and $Q$ using simulation. For each combination of $\beta$ and $Q$, the dual PLP model was solved with the Solver embedded in Microsoft Excel 2007, which gives us $v^{*}(\beta, Q)$. The feasible range of $\beta$ and $Q$ derived from equation (7) and (8) are given in Table 1.

TABLE I. FEASIBLE RANGE OF $\beta$ AND $Q$

\begin{tabular}{|l|l|}
\hline Variation rate $(\beta)$ & Nominal quantity $(Q)$ \\
\hline$[0,1]$ & {$\left[\frac{D_{T}}{T(1+\beta)}, \frac{D_{T}}{T(1-\beta)}\right]$} \\
\hline
\end{tabular}

To make the flexibility analysis of the contract, it is necessary to provide the feasible range of $\beta$ and $Q$ in another way round, where the range of $\beta$ is the function of $Q$. That is, for a fixed value $Q$, all possible $\beta$ values needs to be explore to find the best one to provide the lowest total cost. From Table 1 , it is easily deduced that the range of $\beta$ is $\left[\max \left(1-\frac{D_{T}}{Q \cdot T}, \frac{D_{T}}{Q \cdot T}-1\right), 1\right]$.

\section{A. Input Data}

The input data to the dual $P L P$ model is given in Table 2 and Table 3, where $T=12$ months in a year. Each period is one month.

Table 2 below, represents a one year historical demand (forecast) and the accumulation of the demands for each period. Table 3 shows that the demand is not stationary over the planning horizon.
TABLE II. DEMAND IN A YEAR

\begin{tabular}{|c|c|c|}
\hline $\boldsymbol{t}$ (month) & $\boldsymbol{d}_{\boldsymbol{t}}$ (units) & $\boldsymbol{D}_{\boldsymbol{t}}$ (units) \\
\hline $\mathbf{1}$ & 100 & 100 \\
\hline $\mathbf{2}$ & 100 & 200 \\
\hline $\mathbf{3}$ & 100 & 300 \\
\hline $\mathbf{4}$ & 90 & 390 \\
\hline $\mathbf{5}$ & 110 & 500 \\
\hline $\mathbf{6}$ & 120 & 620 \\
\hline $\mathbf{7}$ & 80 & 700 \\
\hline $\mathbf{8}$ & 70 & 770 \\
\hline $\mathbf{9}$ & 130 & 900 \\
\hline $\mathbf{1 0}$ & 80 & 980 \\
\hline $\mathbf{1 1}$ & 120 & 1100 \\
\hline $\mathbf{1 2}$ & 100 & 1200 \\
\hline
\end{tabular}

Where $\boldsymbol{D}_{\boldsymbol{t}}$ is the accumulated demand.

The holding and shortage costs are stationary through the planning horizon, i.e., $h_{t}=h, s_{t}=s$. The minimum possible $\operatorname{cost}, c_{0}$ and $c_{1}$ are fixed. According to the assumption $\sum_{t=1}^{T} x_{t}=\sum_{t=1}^{T} d_{t}=D_{T}$, we have the total demand, $D_{T}=1200$.

TABLE III. INPUT DATA

\begin{tabular}{|c|c|c|c|c|}
\hline $\boldsymbol{h}(£)$ & $\boldsymbol{s}(£)$ & $\boldsymbol{c}_{\mathbf{0}}(£)$ & $\boldsymbol{D}_{\boldsymbol{T}}$ (units) & $\boldsymbol{c}_{\mathbf{1}}(£)$ \\
\hline 2 & 10 & 10 & 1200 & 0.5 \\
\hline
\end{tabular}

\section{B. Optimisation of the pLP model}

Since computing all the combinations of $\beta$ and $Q$ is exhaustive, and bearing in mind that the convexity of the dual pLP with respect to $\beta$ when $Q$ is fixed and the convexity of the dual pLP with respect to $Q$ when $\beta$ is fixed were theoretically verified (Theorem 1). Decision was made to conduct the optimisation using Equal Interval Search (EIS) Method-which helped narrow the sampling space.

EIS Method is one of the techniques used for finding the extreme value (minimum or maximum of a strictly unimodal function by successively narrowing the range of values inside which the extreme value is known to exist.

The basic idea of this EIS Method to explore all possible solutions of $g(\beta, Q)$ is to explore all the possible value of $Q$ by starting with $Q=0$, then increase the value of $Q$ by 1 each time. With a give value $Q$, we don't need to explore all the values of $\beta$ in $\left[\max \left(1-\frac{D_{T}}{Q \cdot T}, \frac{D_{T}}{Q \cdot T}-1\right), 1\right]$.

Due to the fact that the convexity of the dual pLP with fixed value $\beta$ was theoretically verified, we can apply EIS Method 
to explore limited number of value $Q$ without loss of optimality.

To help simulate the behaviour of $g(\beta, Q)$, a macro -set of VBA codes was written and imbedded in Excel to implement EIS Method.

\section{Optimisation results}

Table 4 gives part of the simulation results, where $Q$ is fixed to 100 , which is the mean of the forecasted demand $\boldsymbol{d}_{\boldsymbol{t}}$ and the value of $\beta$ is explored using EIS method.

TABLE IV. SIMULATION RESULTS FOR $Q=100$

\begin{tabular}{|c|c|c|c|c|}
\hline $\boldsymbol{Q}$ & $\boldsymbol{\beta}$ & $\boldsymbol{V}_{\boldsymbol{h s}}$ & $\boldsymbol{K}_{\boldsymbol{t}}$ & $\boldsymbol{g}(\boldsymbol{\beta}, \boldsymbol{Q})$ \\
\hline 100 & 0.00 & 320 & 12,000 & 12,320 \\
\hline 100 & 0.50 & 0 & 12,300 & 12,300 \\
\hline 100 & 0.51 & 0 & 12,306 & 12,306 \\
\hline 100 & 1.00 & 0 & 12,600 & 12,600 \\
\hline 100 & 0.01 & 300 & 12,006 & 12,306 \\
\hline 100 & 0.25 & 10 & 12,150 & 12,160 \\
\hline 100 & 0.26 & 8 & 12,156 & 12,164 \\
\hline 100 & 0.49 & 0 & 12,294 & 12,294 \\
\hline 100 & 0.02 & 280 & 12,012 & 12,292 \\
\hline 100 & 0.13 & 98 & 12,078 & 12,176 \\
\hline 100 & 0.14 & 84 & 12,084 & 12,168 \\
\hline 100 & 0.24 & 12 & 12,144 & 12,156 \\
\hline 100 & 0.15 & 70 & 12,090 & 12,160 \\
\hline 100 & 0.19 & 30 & 12,114 & 12,144 \\
\hline $\mathbf{1 0 0}$ & $\mathbf{0 . 2 0}$ & $\mathbf{2 0}$ & $\mathbf{1 2 , 1 2 0}$ & $\mathbf{1 2 , 1 4 0}$ \\
\hline 100 & 0.23 & 14 & 12,138 & 12,152 \\
\hline 100 & 0.21 & 18 & 12,126 & 12,144 \\
\hline 100 & 0.22 & 16 & 12132 & \\
\hline & & & & \\
\hline
\end{tabular}

It is noted in Table 4 that the optimal total cost, $g(\beta, 100)=$ 12,140 , is achieved when $\beta=0.20$.

Table 5 provides the simulation results for different $Q$ values. For each fixed value $Q^{F}$, only the $\beta$ value, which can provide minimum $g\left(\beta, Q^{F}\right)$, is kept in one row of Table 5. Due to the size of the paper, we just provide the results of $Q$ in the interval $[90,110]$.
TABLE V. SIMULATION RESULTS FOR DIFFERENT $Q$ VALUES.

\begin{tabular}{|c|c|c|c|c|c|}
\hline $\boldsymbol{Q}$ & $\beta$ & $g(\beta, Q)$ & $\boldsymbol{Q}$ & $\beta$ & $g(\beta, Q)$ \\
\hline $\mathbf{9 1}$ & 0.26 & 12,208 & $\mathbf{1 0 2}$ & 0.22 & 12,150 \\
\hline $\mathbf{9 2}$ & 0.25 & 12,200 & $\mathbf{1 0 3}$ & 0.22 & 12,154 \\
\hline $\mathbf{9 3}$ & 0.24 & 12,192 & $\mathbf{1 0 4}$ & 0.23 & 12,159 \\
\hline $\mathbf{9 4}$ & 0.22 & 12,184 & $\mathbf{1 0 5}$ & 0.24 & 12,163 \\
\hline $\mathbf{9 5}$ & 0.21 & 12,176 & $\mathbf{1 0 6}$ & 0.25 & 12,167 \\
\hline $\mathbf{9 6}$ & 0.21 & 12,169 & $\mathbf{1 0 7}$ & 0.25 & 12,172 \\
\hline $\mathbf{9 7}$ & 0.19 & 12,161 & $\mathbf{1 0 8}$ & 0.26 & 12,176 \\
\hline $\mathbf{9 8}$ & 0.21 & 12,154 & $\mathbf{1 0 9}$ & 0.27 & 12,180 \\
\hline $\mathbf{9 9}$ & 0.20 & 12,147 & $\mathbf{1 1 0}$ & 0.27 & 12,184 \\
\hline $\mathbf{1 0 0}$ & 0.20 & 12,140 & & & \\
\hline
\end{tabular}

The graph of the simulation work in Table 5 is shown in Figure 1.

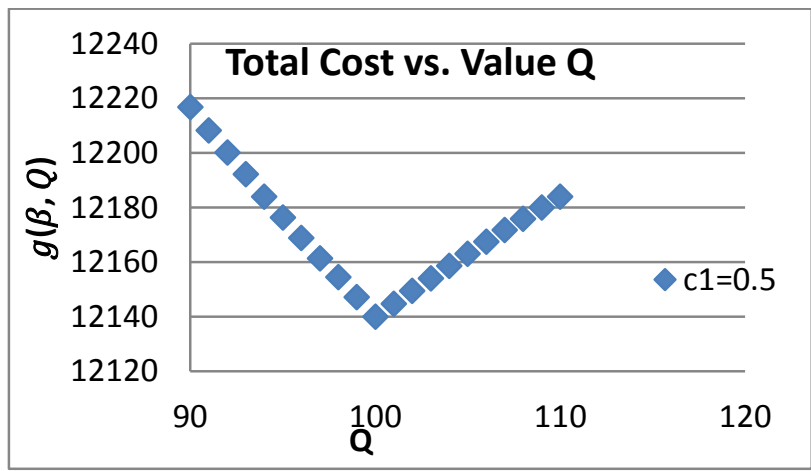

Figure 1. Relationship of total $\operatorname{cost} g(\beta, Q)$ and variable $Q$

It can be seen that $g(\beta, Q)$ is a unimodal function with respect to both $\beta$. In the future, we aim to explore this feature theoretically.

Table 6 below gives the best simulation result for the Data Input provided in section $\mathrm{A}$. The optimum variation rate $(\beta)$, the optimum nominal quantity $(Q)$, the total holding/shortage cost $\boldsymbol{v}(\beta, Q)$ and total cost $g(\beta, Q)$ over the length of the contract are all listed.

TABLE VI. OPTIMUM RESULT FROM SIMULATION WORK

\begin{tabular}{|l|l|c|c|c|}
\hline $\boldsymbol{c}_{\mathbf{1}}=\mathbf{0 . 5}$ & $\boldsymbol{\beta}^{*}$ & $\boldsymbol{Q}^{*}$ & $\boldsymbol{v}^{*}(\boldsymbol{\beta}, \boldsymbol{Q})$ & $\boldsymbol{g}^{*}(\boldsymbol{\beta}, \boldsymbol{Q})$ \\
\hline Optimum & 0.2 & 100 & 20 & 12140 \\
\hline
\end{tabular}

\section{CONCLUSION}

A successful simulation of $p L P$ problem was achieved in this work. The results shown in fig. 1 clearly validate the conclusion that $g(\beta, Q)$ is convex with respect to $\beta$ and $Q$ the theoretical proof of joint convexity of both $\beta$ and $Q$ will be our future research. Also, the trade-off of $h, s, c_{0}$ and $c_{1}$ with respect to the total cost will also be analysed in the future. 


\section{ACKNOWLEDGMENT}

The authors would like to thank the reviewers for their valuable and detailed comments which have greatly improved the presentation of this work.

\section{REFERENCES}

[1] Beamon, B.M, 1998. "Supply chain design and analysis: Models and methods". International Journal of Production Economics, 55, 281294.

[2] Das, S.K., Abdel-Malek, L., 2003. "Modelling the flexibility of order quantities and lead times in supply chain". International journal of production Economics 85 (2), pp171-181

[3] Gerard P. Cachon, 2003. "Supply Chain Coordination with Contracts"

[4] Hohn, M.I., 2010. "Relational supply contracts, Lecture Notes in Economics and Mathematics Systems", Springer-Verlag Berlin Heidelberg,

[5] Jing Checn, 2012. "Contracting in a newsvendor problem". Journal of Modelling in Management", Vol. 7 No. 3, pp.242-256

[6] Keely L. Croxton, Sebastian J. Garcia-Dastugue, Douglas M. Lambert and Dale S. Rogers, 2001. "The supply Chain Management Process" The international Journal of Logistics Management, pp-01

[7] Keen, S.E., Kanchanapiboon, A., Das, S.K., 2010. "Evaluating supply chain flexibility with order quantity constraints and lost sales", International journal of Production Economics 126, pp 181-188.

[8] Kim, W.S., 2011. "Order quantity flexibility as a form of customer service in a supply chain contract model", Flexible Services and Manufacturing Journal 29(3), pp 290-315.

[9] Knoblich, K., Ehm, H., Heavey, C., Williams, P., 2011. "Modelling Supply Contracts in Semiconductor Supply Chains", IEEE

[10] Lariviere, M.A., 1999. "Supply chain contracting and coordination with stochastic demand”. In: Tayur, S., Magasine, M., Ganeshan, R. (Eds.), Quantitative Models for supply chain management. Springer.

[11] Liu Bein-li and MA Wen-hui, 2008. "Application of Quantity flexibility contract in perishable products supply chain coordination", IEEE

[12] Nihar Sahay and Marianthi G. Ierapetritou, 2013. "Centralized Vs. Decentralized Supply Chain Management Optimization"

[13] Sethi, S.P., Yan, H., Zhang, H., 2004. "Quantity flexibility contracts: optimal decision with information updates, Decis”. Sci. 35 691-712.

[14] Tsay, A.A, 1999. "Quantity flexibility contract and supplier-customer incentives”, Management science 45 (10), pp1339-1358. 